\title{
The response of the accommodation system to digital and print images
}

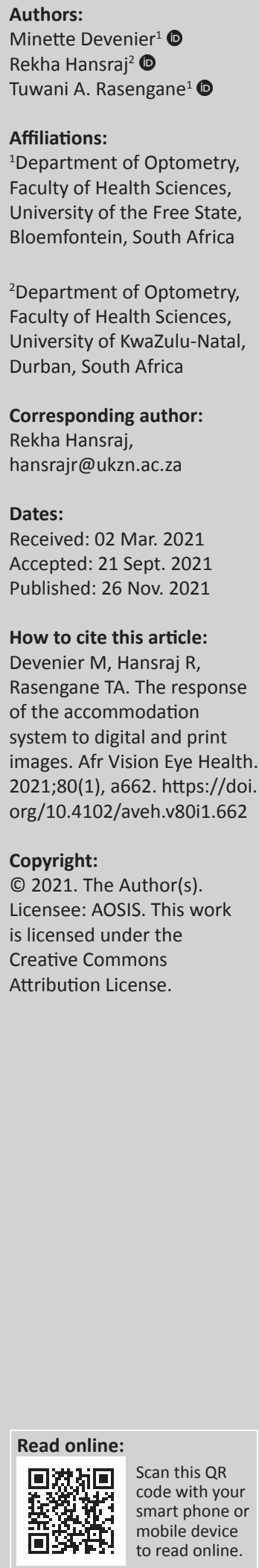

Background: The transition from traditional print medium to a digital medium may affect the accommodative response (AR) because of the differences in the characteristics of the targets viewed.

Aim: This study investigated how the accommodation system responded to targets displayed on a tablet computer compared to that on paper.

Setting: The study was conducted amongst students at a university in South Africa.

Methods: Using a quantitative, cross-sectional study the AR, amplitude of accommodation (AA), and accommodative facility (AF) were assessed with a target on an iPad and a paperbased one on a non-probability sample of 30 university students. Data was analysed using descriptive statistics and Bland Altman plots.

Results: The median AR with a tablet was +0.25 dioptre (D) compared to $+0.21 \mathrm{D}$ with the paper-based target. This difference was neither statistically nor clinically significant. The median AA with a tablet computer target was $10.59 \mathrm{D}$ and $9.85 \mathrm{D}$ with a paper-based target. While this difference was statistically significant $(p=0.002)$, Bland Altman analysis revealed comparable measurements with both types of targets. Both Wilcoxon Signed Ranks Test $(p=0.462)$ and Bland Altman analysis found comparable medians for AF obtained with a target on paper (7.67 cycles per minute [cpm]) and a target on the tablet computer $(7.17 \mathrm{cpm})$ to be comparable.

Conclusion: The accuracy, strength and flexibility of accommodation were comparable for tablet computer and paper-based targets.

Keywords: accommodation; accommodation response; amplitude of accommodation; accommodation facility; iPad; paper.

\section{Introduction}

Computer technology has evolved considerably, and rapidly, over the past decades with the most recent advance being the transition from desktops to laptops and now to even smaller mobile devices, one of which is a tablet computer. A tablet computer is a mobile personal computer with a touch sensitive screen that can be operated by a stylus, pen or finger, with the iPad being a tablet computer that was introduced by the multinational technology company Apple Inc. in the year 2010. ${ }^{1}$ The use of mobile computing, which makes it possible for users to meet their computing demands in all instances and places, has become ubiquitous over the course of the last two decades. ${ }^{2,3}$ It was accurately predicted that the use of tablet computers would take over desktop usage in $2015,{ }^{4}$ and this trend has extended from the corporate to the education sector. Results show that the use of tablet computers in tertiary education, has increased twice as much even in the course of a single year ${ }^{5}$ with teaching and learning having become reliant on digital learning platforms. The popularity of these devices can be attributed to them being reasonably priced, environmentally friendly, and enabling an entire library to be carried in a backpack. ${ }^{6}$

Despite the numerous advantages, on the flip side, computer-based tasks have been associated with computer vision syndrome (CVS), also known as digital strain, which Rosenfield et al. ${ }^{7}$ defined as 'the combination of eye and visual problems associated with the use of computers'. The most prevalent ocular symptoms found in office workers with this syndrome included blurred vision, asthenopia and dry eyes. ${ }^{8}$ It was postulated early on that this condition may occur because of the visual demand of the computer task exceeding the visual capabilities of the observer, and this may interfere with reading and near work performance and subsequently efficiency of the user. ${ }^{9,10}$ Interestingly, Chu et al. ${ }^{11}$ found that symptoms of CVS were 
significantly worse following sustained computer use rather than reading printed text. Being a device that is held at a near working distance, visual performance and comfort is heavily reliant on accommodation and convergence. However, there has been limited research on whether these visual functions respond differently for computer displayed targets compared to paper-based ones. The focus of this article will be on the accommodation system and how it responds to near point tasks with digital and paper-based visual images or texts.

Accommodation is defined broadly as the ability of the eyes to alter its refractive powers to focus on objects positioned closer than infinity, thereby allowing for the target to be seen clearly.12 The accommodation system can be evaluated in terms of the accuracy of the response (lag or lead), strength (amplitude) and flexibility (facility). ${ }^{13}$ The stimulus to accommodation is retinal blur that is influenced by object characteristics including spatial arrangement and contrast, and various parameters like working distance, text sizes, and gaze angles, which are reportedly different when comparing the tablet computer to paper-based text. ${ }^{14}$ Furthermore, paper-based targets have lesser reflections, better contrast and thus provide a sharper image than that on a computer screen. Digital text consists of thousands of tiny pixels whose contrast and stability may affect visual performance. ${ }^{15}$ Sheedy and Shaw-McMinn ${ }^{16}$ reported that almost a third of the patients having CVS had an accommodative problem, however, information on how the accommodation system is affected with the use of digital devices remains poorly understood. ${ }^{7}$

Accuracy of accommodation measured in terms of lag of accommodation have been found to differ for targets on a computer monitor compared to those on paper, often with a larger lag measured with the computer target. ${ }^{17,18,19}$ While the amplitude of accommodation (AA) achievable with a digital target compared to a paper-based target has not been previously investigated, studies have found this component to decrease with prolonged computer use. ${ }^{20,21}$ Similarly, even though accommodative facility (AF) has not been compared between the two mediums, it has been the most common binocular vision anomaly observed in CVS patients through a retrospective review of clinical records ${ }^{22}$ with Rosenfield et al. ${ }^{23}$ reporting an increase in binocular AF on completion of a computer task.

Many of the previous studies in this area have been done at least a decade ago, and since then there have been major developments in the technology of computerised devices including character generation. The majority of previous studies have studied the accuracy of the accommodative response (AR) in terms of measurement of the lag of accommodation only. However, a better assessment would ideally include the strength of the AR, that is, AA and flexibility of the accommodative system expressly, AF. Furthermore, previous studies on accommodative accuracy with computerised targets have been done on devices that have much larger screen sizes and are used at a fixed working distance which is larger than the more popular mobile devices used nowadays including the iPad. Only one other study $^{3}$ was found that attempted to compare the AR to targets displayed on ink on paper, an e-ink reader and an liquid crystal displays (LCD) device. This study however focussed on the AR with particular emphasis on its relation to pupil size, but did not report on AA or AF.

Even though the popularity of tablets has increased, studies addressing the question on how the use of these devices can comfortably and efficiently be incorporated into everyday life are still lacking. ${ }^{24}$ The current study will provide information on the response of the accommodative system in terms of accuracy, strength and flexibility when using a tablet computer such as an iPad in comparison to conventional paper-based targets. These findings may also provide a guide to the appropriate functional aspects that should be examined in the eye testing routine, as well as the management plan particularly for patients working on computer devices such as iPads. Furthermore, clinicians will be aware of possible effects of interchanging electronic and paper-based targets on clinical test measurements and subsequently any clinical management based on them.

\section{Methodology}

This study utilised a quantitative, cross-sectional, descriptive, design and was conducted on a non-probability sample of 30 university students selected by convenience. The sample included participants of any gender, race and between the ages of 18 years and 25 years who were able to obtain aided or unaided distance and near Snellen visual acuity of $6 / 6$ or better. The presence of a heterotropia, ocular or systemic disease formed the exclusion criteria for this study. The tablet computer that was used to conduct the study was an iPad Mini with Retina Display ${ }^{\mathrm{TM}}$. Retina display is a name given by the Apple company to products having higher pixel density which reduces the likelihood of being able to detect individual pixels and thereby reducing digital eye strain. A near point card was used for the paper-based target and the size, font and contrast of the targets were similar when displayed either on the iPad or near card. In addition, a reading stand was used to ensure that both the iPad and paper target were placed at the same distance from the participant.

Three tests assessing different aspects of the AR were performed. The measurement of the accuracy of the AR was taken using the fused cross cylinder $( \pm 0.50$ dioptre [D]) technique. The AA measurement was taken using the pushup-to-blur method, and AF with the lens rock method using \pm 2 D flippers. Each of the aforementioned techniques provided binocular subjective measurements and were done with the distance prescription on wherever relevant. They were performed according to established procedure ${ }^{25}$ and constant room illumination was monitored with an iPad Mini application called Megaman LuxMeter. The order in 
which the targets and tests were administered was randomised to minimise both the learning effect and the effect of fatigue. Every test, using each of the targets, was repeated three times and for each test the average value was recorded.

Descriptive and inferential statistics were used in the analysis of the results using Statistical Analysis Software (SAS) version 9.4 and the Statistical Package for Social Sciences (SPSS) version 27, under the guidance of a statistician. The Fischer Exact test and Bland and Altman analyses were also conducted wherever relevant or possible. The tenets of the Declaration of Helsinki were adhered to in all aspects of the study.

\section{Ethical considerations}

Ethical approval for this study was received from the Biomedical Ethics and Research Committee at the University of KwaZulu-Natal (BE010/15).

\section{Results}

The mean age of the participants was $20.16( \pm 1.57)$ years, however as $40 \%$ of them were 19 years of age, the median age was calculated and found to be 19.5 (interquartile range $[I Q R]=19-22)$ years. The majority $(70 \%)$ of the participants were male. All participants could be classified as low ametropias, with the majority (88.5\%) having a low degree of astigmatism and $50.5 \%$ being myopic. The mean heterophoria for the sample was found to be $4.06 \pm 4.38 \mathrm{pd}$ exophoria as determined with the Von Graefe method. Table 1 provided a summary of the results (medians for iPad and paper target) for each of the accommodative components assessed. The results for the detailed analysis of each component follows.

\section{Accuracy of accommodative response}

The accuracy of the AR to the accommodative demand was measured using the fused cross cylinder method, thus the findings are given in dioptres (D). The median AR when using a target on the iPad was $+0.25 \mathrm{D}(\mathrm{IQR}=0.08-0.41)$ as compared to a slightly lower median of $+0.21 \mathrm{D}(\mathrm{IQR}=$ $0.00-0.35$ ) found when using the target on paper. As the data for this aspect was not normally distributed, the difference was further analysed using the Wilcoxon Signed Ranks Test, and was found to be not statistically significant $(p=0.180)$. This difference in the medians $(0.04 \mathrm{D})$ is also not clinically significant being less than the lowest unit of

\begin{tabular}{lcccc} 
TABLE 1: Summary of medians for the accommodative components assessed. \\
\hline Accommodative component & $N$ & \multicolumn{3}{c}{ Results (median) } \\
\cline { 3 - 6 } & & iPad & Paper & $\begin{array}{c}\text { Wilcoxon } \\
\text { signed-ranks test } \\
\boldsymbol{p} \text {-value }\end{array}$ \\
\hline Accommodative response & 30 & $+0.25 \mathrm{D}$ & $+0.21 \mathrm{D}$ & 0.180 \\
Amplitude of accommodation & 30 & $10.5 \mathrm{D}$ & $+9.85 \mathrm{D}$ & $0.002^{*}$ \\
Accommodative facility & 30 & $7.17 \mathrm{cpm}$ & $7.67 \mathrm{cpm}$ & 0.462 \\
\hline
\end{tabular}

$\mathrm{D}$, dioptre; cpm, cycles per minute.

*, Statistically significant at a $95 \%$ level of confidence. dioptric power of 0.25 D. A Bland Altman plot was not generated for this component of accommodation as the mean differences between the iPad target readings and the paper-based ones were not normally distributed. The Fisher Exact test was carried out to investigate the relationship between refractive error and the AR to targets on paper and iPad. No statistically significant relationship was found on the iPad target $(p=1.0)$ or with the paper target $(p=0.26)$, meaning that the AR of the participants was not dependent on the refractive error.

\section{Amplitude of accommodation}

The AA was measured using the push-up-to-blur method; thus, the measurements were taken in $\mathrm{cm}$ and then converted into D. The data for this aspect was not normally distributed. The median AA measured when using a target on the iPad was 10.59 D (IQR = 9.06-11.72) as compared to a lower median of $9.85 \mathrm{D}(\mathrm{IQR}=8.30-10.76)$ when using a target on paper. While the Wilcoxon signed-ranks test for related samples found the difference to be statistically significant ( $p=0.002)$, further comparison done using Bland Altman analysis (Figure 1) indicated differently. The difference in means for only two subjects were found to lie outside the $95 \%$ limits of agreement indicating good comparability of the findings with the target on the iPad and on paper. The Fisher Exact test was done to investigate if the AA measurements done on the paper and iPad targets were dependent on the refractive error profile of the participants. A statistically non-significant relationship was found with the iPad $(p=1.0)$ and the paper $(p=1.0)$, meaning that the AAs for the participants was not dependent on the refractive profile.

\section{Accommodative facility}

The lens rock method was used to determine the binocular $\mathrm{AF}$ and the measurements were recorded in cpm. The median for measurements obtained with a target on paper of 7.67 cycles per minute $(\mathrm{cpm})(\mathrm{IQR}=3.75-9.41)$ was marginally higher than that for measurements taken with a target on the iPad which was $7.17 \mathrm{cpm}(\mathrm{IQR}=3.50-9.58)$. As the differences in the means for both the components were normally

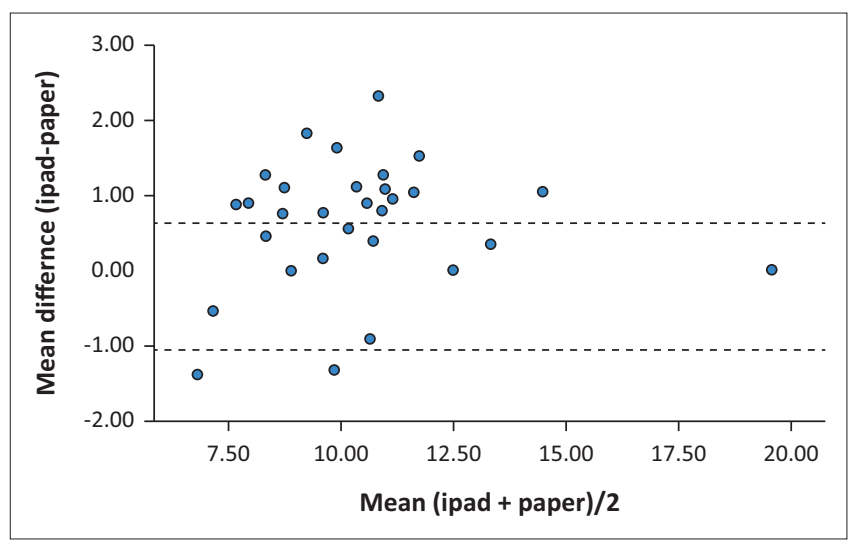

FIGURE 1: Bland Altman plot for mean differences between the measured amplitude of accommodation to targets on the iPad and paper-based targets. 


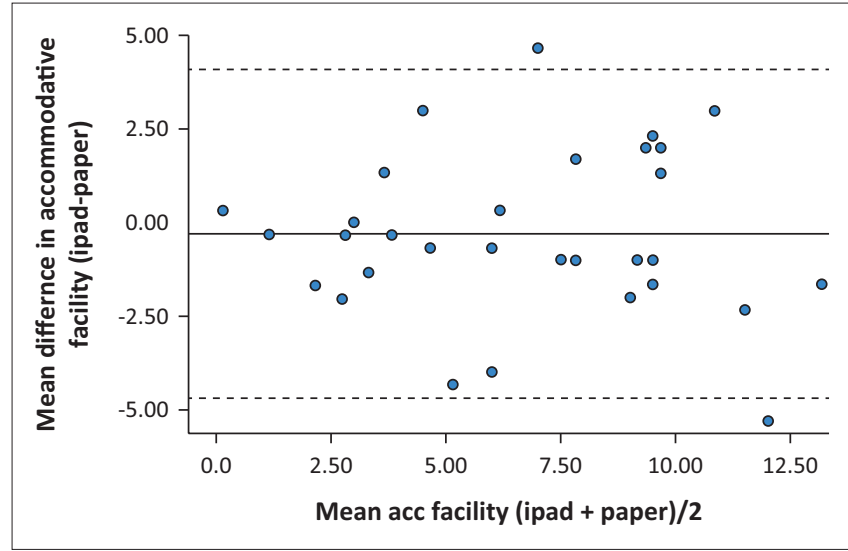

FIGURE 2: Bland Altman plot for mean differences between the measured accommodative facility to targets on the iPad and the paper-based targets.

distributed, further analysis was conducted using the Bland Altman plot (Figure 2). It is noted that majority of the points lie within the $95 \%$ limits of agreement, indicating that the AF measurements taken with the iPad compared well with that obtained with the paper-based target. No statistically significant difference between the readings was noted with the Wilcoxon Signed Ranks Test $(p=0.462)$. The difference was also not regarded as being clinically significant. The Fisher Exact test was carried out to investigate the relationship between the AFs measured on a paper and iPad target with the refractive error profile of the participants. A statistically insignificant relationship was calculated on both the iPad $(p=0.12)$ and the paper $(p=1.0)$ based targets.

\section{Discussion}

Accommodation was assessed in terms of the AR, AA and AF, using an iPad target and a paper target, as these are the parameters that are useful in assessing the accommodative system and are active during near work. The AR was found to indicate a lag of accommodation with both the iPad and paperbased targets. In both cases however, the lag of accommodation was within the acceptable range $(+0.50 \mathrm{D} \pm 0.50) .{ }^{25}$ The median AR when using a target on the iPad was slightly lower that that found using the target on paper, however this difference was neither statistically nor clinically significant. The Bland Altman plot also indicated good comparison of the AR with the target on the iPad and on paper.

Even though studies by Sorkin, Reich and Pizzimenti ${ }^{26}$ and Penisten et al. ${ }^{18}$ used an objective method that is dynamic retinoscopy, instead of the subjective method used in the current study, to assess the AR to targets on a video display terminal to printed texts, they reported a higher lag of accommodation to the digital target. Penisten et al. ${ }^{18}$ also found the same difference in lag of accommodation of $0.04 \mathrm{D}$ between the targets which was also not statistically significant. In contrast, Wick and Morse ${ }^{17}$ and Ferreira, Lira and Franco ${ }^{27}$ found the higher lag for a video display target compared to printed material to be statistically significant. They attributed their findings to differences in spatial frequency characteristics of printed text and pixel letters. The latter two studies though measured the AR with an autorefractor in which examiner influence is negligible compared to dynamic retinoscopy used in other studies and the subjective method used in the current study. Furthermore, in both the studies ${ }^{17,27}$ the video display screens were much larger than the current study (1517 inches versus 7.9 inches with the iPad mini). The screen size does appear to influence the accommodative accuracy, as a more recent study in 2014 recorded a lag of accommodation when reading from an iPod which had a 4 -inch screen, to be significantly higher to that measured when reading from paper. ${ }^{19}$ In addition, the working distances of devices used in previous studies, being desk based, have often been longer than the $40 \mathrm{~cm}$ working distance used in the current study which may also account for differences in findings for ARs. ${ }^{28}$ Notably though, the viewing distance with the iPod in the study by Hue, Rosenfield and Sae ${ }^{19}$ was $33 \mathrm{~cm}$ compared to the $40 \mathrm{~cm}$ working distance in the current study. The findings of the current study are however, comparable to those of a more recent study ${ }^{3}$ which utilised devices of similar characteristics and also reported no significant differences in mean ARs found between an e-ink device and paper, or between paper and an LCD device. The ARs were however, measured objectively.

The AA measured with the iPad target was $0.74 \mathrm{D}$ higher (difference in the medians) than that measured with a paper-based target, in the current study. While the Wilcoxon Signed Ranks Test found the difference to be statistically different, Bland Altman analysis, which has been touted as a better method for comparative studies, revealed comparable AA measurements irrespective of the target being on the iPad or paper. A marginally higher median with the iPad target could imply a slightly closer working distance compared to the paper target. This is particularly relevant as Vasta $^{29}$ reported a mean working distance of $36.2 \mathrm{~cm}$ in adults using hand-held electronic devices, in contrast to $40 \mathrm{~cm}$ when using a hard-copy text, even if the text used was similar in style and size.

The findings of the current study for AA are in keeping with the postulation by Blehm et al. ${ }^{30}$ that VDT work can result in a small, but temporary myopic shift. Excessive stimulation and use of accommodation have also been linked to the progression of myopia, thus myopia control strategies have aimed at relaxing accommodation during near work in an attempt to slow down the progression of myopia. Hence, the use of an iPad may be stimulating more accommodation, and must be taken into consideration when managing iPad users with vision correction.

The higher AA measured with the iPad target is however contrary to the assertion by Wimalsundera ${ }^{31}$ that pixel targets lack sharp edges and thus are expected to create an under stimulation of accommodation. No previous study was found that investigated AA for electronic versus printed targets; thus no further direct comparisons for AA could be made. However, Gur et al. ${ }^{20}$ compared accommodation and convergence in visual display unit (VDU) workers to controls, and reported that the AA of VDU workers decreased by 
around $0.69 \mathrm{D}$ when compared to the control after 4 days of VDU use. Objective measurements of both aspects were taken by Gur et al. $^{20}$ at the beginning of a 4-day working week and again at the end of the week in contrast to the onceoff subjective measurement of AA in the current study. Notably, the study by Gur et al..$^{20}$ was performed over two decades ago when cathode ray tube (CRT) screens were used which had low refresh rates relative to the LCD used by newer devices. The critical fusion frequency of the human eye is reportedly $30 \mathrm{~Hz}$ to $50 \mathrm{~Hz}$, ${ }^{31}$ while the refresh rate of iPad is around $120 \mathrm{~Hz}$ thus making it indistinguishable to the eye hence expectation of better visual performance than with older devices. However, Menozzi and Naepflin ${ }^{32}$ reported no difference in visual performance assessed in terms of visual search times with CRT displays versus LCD displays indicating that the two may be comparable in terms of demands on the visual system.

Comparable measurements were also obtained with both the target on paper and the iPad for AF, which were neither statistically nor clinically significantly different. This component of accommodation is important to assess as Sheedy and Parsons ${ }^{22}$ found accommodative infacility to be the most common binocular vision anomaly in subjects with CVS. No other similar comparative investigation was found in previous studies, but Rosenfield et al. ${ }^{23}$ reported a significant increase in binocular AFs after subjects had completed a computer task. Therefore, while differences in the AR may have been anticipated because of the nature of the computerised targets in comparison to paper-based ones, newer technology including higher density pixel screens might have targets with better defined edges resulting in less eye strain as previously found, and thus minimal effects on ocular accommodation. This may be the reason for the insignificant change in AR found with the paper target compared to the iPad target.

No relationship between the accommodative aspects assessed and refractive error was found in this study, irrespective of the measurement being with the iPad target or target on paper. This is in contrast to the findings of Hinkley et al. ${ }^{13}$ who found subjects with high hyperopia to have greater accommodative lags. However, the range of refractive errors included in that study was much larger than the current study which is a limitation of the current study. Other limitations of the current study include a relatively small sample that was not screened for normal accommodation function prior to inclusion. This study has however assessed accommodation in terms of its accuracy (AR), strength (AA) and speed (AF). While AR has largely been the focus of previous studies that have compared measurements with digital and paper-based targets, minimal to no information is available on $\mathrm{AA}$ and $\mathrm{AF}$ neither on computer devices nor in comparison with paperbased targets. This study thus provides new information in this respect. This study has also utilised a mobile computing device that is currently being used while previous studies have been primarily on desk-top devices with larger screen sizes and lower refresh rates.

\section{Conclusion}

The current study has thus provided information regarding the accommodation system response to digital targets on an iPad in comparison to that of paper-based targets. The importance of this assessment relates to the widespread use of computerised devices, particularly mobile computing devices, in economic, education and social sectors, as well as increasing popularity in the health sector. Optometric assessments, in particular, near vision assessments focussed on the accommodative system, are still often conducted using paper-based targets, the findings of which forms the basis of the management strategy for the patient including vision correction. The findings of the study indicate that the clinical assessment of accommodation using the conventional near vision card, and management thereof, should still be applicable to computerised near tasks and activities.

\section{Acknowledgements Competing interests}

The authors declare that they have no financial or personal relationships that may have inappropriately influenced them in writing this article.

\section{Authors' contributions}

M.D. was the principal investigator and involved in the conceptualisation, data collection and analysis, and writing of the paper. R.H. and T.A.R. were the supervisors and were also involved in the conceptualisation, data analysis and writing of the paper.

\section{Funding information}

This research received no specific grant from any funding agency in the public, commercial or not-for-profit sectors.

\section{Data availability}

The data that support the findings of this study are available on request from the corresponding author, R.H..

\section{Disclaimer}

The views and opinions expressed in this article are those of the authors and do not necessarily reflect the official policy or position of any affiliated agency of the authors.

\section{References}

1. Atkinson P. A bitter pill to swallow: The rise and fall of the tablet computer. Des Issues. 2008;24(4):3-25. https://doi.org/10.1162/desi.2008.24.4.3

2. Sears A, Arora R. Data entry for mobile devices: An empirical comparison of novice performance with jot and graffiti. Interact Comput. 2002;14(5):413-433. https:// doi.org/10.1016/S0953-5438(01)00060-1

3. Cisarik PM, Nguyen J. Comparison of accommodative responses to e-Ink vs. LCD vs. Standard Ink on hard copy. OVP. 2019;7(5):269-281. 
4. Haselton T. More mobile internet users than wireline users in the U.S. by 2015 [homepage on the Internet]. Framingham, MA: International Data Corporation 2011 [cited 2021 Feb 3]. Available from: https://bgr.com/2011/09/12/u-s-touse-mobile-internet-more-than-wireline-by-2015-idc-says/

5. Chen B, De Noyelles A. Exploring students' mobile learning practices in higher education. EDUCAUSE Review [serial online]. 2013 [cited 2014 Mar 18] Available from: https://er.educause.edu/articles/2013/10/exploring-studentsmobile-learning-practices-in-higher-education

6. Benedetto S, Drai-Zerbib V, Pedrotti M, et al. E-readers and visual fatigue. PLoS One [serial online]. 2013;8(12):e83676. https://doi.org/10.1371/journal. pone.0083676

7. Rosenfield M, Hue J, Huang R, et al. Uncorrected astigmatism and computer vision syndrome. Invest Ophthalmol Vis Sci. 2011;52(14):2815.

8. Portello J, Bababekova $\mathrm{Y}$, Rosenfield $\mathrm{M}$, et al. Computer-related visual symptoms in office workers. Ophthalmic Physiol Opt. 2012;32(5):375-382. https://doi. org/10.1111/j.1475-1313.2012.00925.x

9. Anshel J, editor. Visual ergonomics handbook. Boca Raton, FL: Taylor and Francis Group; 2005.

10. Grisham DJ, Sheppard MM, Tran WU. Visual symptoms and reading performance. Optom Vis Sci. 1993;70(5):384-391. https://doi.org/10.1097/00006324-19930500000008

11. Chu C, Rosenfield M, Portella JK, et al. A comparison of symptoms after viewing text on a computer screen and hard copy. Ophthalmic Physiol Opt. 2011;31(1): 29-32. https://doi.org/10.1111/j.1475-1313.2010.00802.x

12. Yanoff M, Duker JS, Augsburger JJ, et al. Ophthalmology. 3rd ed. Edinburgh: Mosby Elsevier; 2009.

13. Hinkley S, Iverson-Hill S, Haack L. The correlation between accommodative lag and refractive error in minors under 18. Austin J Clin Ophthalmol. 2014;1(2):1-5.

14. Bababelova $\mathrm{Y}$, Rosenfield $\mathrm{M}$, Hue JE, et al. Font size and viewing distance of handheld smartphones. Optom Vis Sci. 2011;88(7):795-797. https://doi. org/10.1097/OPX.0b013e3182198792

15. National Research Council (US) Panel on Impact of Video Viewing on Vision of Workers; National Research Council (US) Committee on Vision. Video displays, work, and vision. Washington, DC: National Academies Press (US); 1983.

16. Sheedy JE, Shaw-McMinn PG. Diagnosing and treating computer-related vision problems. Amsterdam: Butterworth-Heinemann; 2003.

17. Wick B, Morse S. Accommodative accuracy to video display monitors. Optom Vis Sci. 2002;79(12s):218. https://doi.org/10.1097/00006324-20021200100413
18. Penisten DK, Goss DA, Philpott G, et al. Comparison of dynamic retinoscopy measurements with a print card, a video display terminal, and a PRIO system tester as test targets. Optometry. 2004;75(4):231-240. https://doi.org/10.1016/ tester as test targets. Op

19. Hue JE, Rosenfield M, Sae G. Reading from electronic devices versus hard copy. Work. 2014;47(3):303-307. https://doi.org/10.3233/WOR-131777

20. Gur S, Ron S, Heicklen-Klein A. Objective evaluation of visual fatigue in VDU workers Occup Med. 1994;44(4):201-204. https://doi.org/10.1093/occmed/44.4.201

21. Yeow PT, Taylor SP. Effects of long-term visual display terminal usage on visual functions. Optom Vis Sci. 1991;68(12):930-941. https://doi.org/10.1097/ 00006324-199112000-00004

22. Sheedy JE, Parsons SD. The video display terminal eye clinic, clinical report. Optom Vis Sci. 1990;67(8):622-626. https://doi.org/10.1097/00006324-199008000-00012

23. Rosenfield $M$, Gurevich $R$, Wickware $E$, et al. Computer vision syndrome: Accommodative and vergence facility. J Behav Optom. 2010;21(5):119-122.

24. Ozok A, Benson D, Chakraborty J, et al. A comparative study between tablet and laptop PCs: User satisfaction and preferences Int Hum-Comput Int. 2008;24(2):329-352. https://doi.org/10.1080/10447310801920524

25. Carlson NB, Kurtz D. Clinical procedures for ocular examination. 4th ed. New York, NY: McGraw-Hill; 2004.

26. Sorkin RE, Reich LN, Pizzimenti J. Accommodative response to PRIO computer vision tester versus printed text. Optometry. 2003;74(12):782-786

27. Ferreira A, Lira M, Franco S. Accommodative and convergence response to computer screen and printed text [homepage on the internet]. Proceedings of SPIE - The International Conference on Applications of Optics and Photonics; 2011 Jul 26 [cited 2014 Mar 18]. Available from: https://www.researchgate.net/ publication/253074820_Accommodative_and_Convergence_Response_to Computer_Screen_and_Printed_Text

28. Tosha C, Borsting E, Ridder WH, et al. Accommodative response and visual discomfort. Ophthalmic Physiol Opt. 2009;29(6):625-633. https://doi. org/10.1111/j.1475-1313.2009.00687.x

29. Vasta S. Ergonomics research aims to help reduce visual demands of computer use. Primary Care Optometry News. 2008;13(4):24.

30. Blehm C, Vishnu S, Mitra AS, et al. Computer vision syndrome, a review. Surv Ophthalmol. 2005;50(3):253-262. https://doi.org/10.1016/j.survophthal.2005.02.008

31. Wimalasundera S. Computer vision syndrome. Galle Med J. 2006;11(1):25-29. https://doi.org/10.4038/gmj.v11i1.1115

32. Menozzi M, Naepflin U. CRT versus LCD: Effects of refresh rate, display technology and background luminance in visual performance. Displays. 2001;22:79-85. https://doi.org/10.1016/S0141-9382(01)00054-3 